\title{
Asile, immigration : réconcilier les Droits de l'homme et ceux du citoyen
}

\section{Luc Legoux}

\section{(2) OpenEdition}

1 Journals

\section{Édition électronique}

URL : https://journals.openedition.org/remi/2822

DOI : $10.4000 /$ remi.2822

ISSN : $1777-5418$

Éditeur

Université de Poitiers

\section{Édition imprimée}

Date de publication : 1 juillet 2006

Pagination : 95-103

ISBN : 2-911627-42-3

ISSN : 0765-0752

\section{Référence électronique}

Luc Legoux, " Asile, immigration : réconcilier les Droits de l'homme et ceux du citoyen », Revue européenne des migrations internationales [En ligne], vol. 22 - n² | 2006, mis en ligne le 01 juin 2009, consulté le 14 avril 2022. URL : http://journals.openedition.org/remi/2822 ; DOI : https://doi.org/ 10.4000/remi.2822

Ce document a été généré automatiquement le 14 avril 2022.

(C) Université de Poitiers 


\title{
Asile, immigration : réconcilier les Droits de l'homme et ceux du citoyen
}

\author{
Luc Legoux
}

1 Il y a vingt ans la forte croissance du nombre des demandeurs d'asile en Europe transformait la défense des droits humains en problème migratoire. Depuis, au fil des ans, la question de l'asile est progressivement devenue centrale dans la politique migratoire européenne et elle a fortement contribué à la judiciarisation croissante de la gestion des migrations et des frontières. Aujourd'hui, résultat d'une politique répressive efficace et non d'une amélioration du respect des droits humains dans le monde, le nombre de demandes d'asile baisse fortement dans l'Union européenne.

2 En France, le thème de l'immigration "choisie " opposée à l'immigration "subie » développé par le ministre de l'Intérieur Nicolas Sarkozy est révélateur des priorités du gouvernement en matière de politique d'accueil. Dans le projet de loi en cours, l'immigration « choisie » est celle des migrants apportant les « compétences et talents » dont notre pays a besoin, l'immigration « subie » rassemble l'immigration familiale, les régularisations et l'asile. La caractéristique commune de ces trois modes d'accès à un titre de séjour est un rapport étroit avec les droits humains. L'immigration familiale, que le président Giscard d'Estaing avait voulu interdire, a été imposée par le Conseil d'État au nom du droit fondamental à mener une vie familiale normale (arrêt Gisti 1978) ; les régularisations sont faites pour accorder des droits à des personnes que l'on ne peut expulser; chercher asile est un droit humain fondamental reconnu dans de nombreux textes dont la déclaration universelle des droits de l'homme de 1948. L'immigration dite "subie " à laquelle s'oppose le ministre de l'Intérieur est celle qu'impose le respect des droits humains. Comment en est-on arrivé là dans la patrie des droits de l'homme? 


\section{L'asile victime des charters ministériels}

Le 18 octobre 1986, un charter de 101 Maliens organisé par le ministre de l'Intérieur Charles Pasqua est, involontairement, à l'origine des premiers détournements massifs de la procédure d'asile. Par ce coup médiatique, 101 n'est pas un chiffre innocent, le ministre déclare vouloir envoyer un signal fort aux ressortissants des pays d'origine : ne venez plus clandestinement ou vous serez impitoyablement expulsés. Les mois suivants, la demande d'asile malienne décuple. L'arrivée du charter à Bamako a-t-elle eu l'effet inverse de celui escompté et suscité une envie subite et massive de venir demander l'asile en France ? Bien sûr que non ; la causalité entre le charter du ministre et l'accroissement de la demande malienne est réelle mais n'a rien à voir avec une immigration nouvelle. Les Maliens présents irrégulièrement en France de longue date et jusqu'alors relativement tolérés ont pris peur. Pour éviter d'être obligés de retourner au Mali, ils ont cherché un moyen d'échapper aux expulsions et ont trouvé la demande d'asile.

Il faut dire qu'à l'époque la faiblesse des moyens accordés à l'office français de protection des réfugiés et des apatrides (Ofpra) et à la Commission des recours (CRR), se traduisait par de très longs délais de traitement, environ 2 à 3 ans devant l'ofpra suivis d'une période au moins aussi longue devant la juridiction de recours. Demander l'asile revenait donc à obtenir une longue régularisation de fait, avec de surcroît, jusqu'à la fin de 1991, le droit au travail. De plus, sous l'effet de l'accroissement de la demande, les délais de traitement s'allongeaient encore, ce qui accroissait d'autant l'intérêt d'une demande d'asile non fondée sur des craintes de persécutions.

Bien entendu, les Maliens ne sont pas les seuls à avoir utilisé la procédure d'asile pour se maintenir en France. Progressivement toutes les nationalités y ont eu recours ce qui induit une grande confusion entre les demandes fondées sur des craintes réelles de persécution et les demandes déposées pour échapper aux expulsions. C'est ainsi que lors du pic de 1989 on enregistrait 61000 demandes dont 27000 Turcs mêlant des Kurdes fuyant des persécutions en Turquie et des immigrants illégaux turcs installés de longue date. Une analyse statistique du devenir des demandes de 1990 a ainsi montré que malgré des critères de reconnaissance très restrictifs, un tiers des demandeurs turcs, essentiellement des Kurdes, ont été reconnus réfugiés à l'issue de la procédure (Legoux, 1995).

6 Cet effet pervers d'une politique répressive ciblée sur les immigrés illégaux n'est pas resté inconnu du gouvernement, Dès le rapport d'activité de l'ofpra de l'année 1986, le phénomène de vases communicants entre la présence illégale de longue date et la demande d'asile suite au charter pour le Mali est décrit en détail. En 1986 comme en 2006, cette connaissance du phénomène n'est pas prise en compte par le gouvernement. La lutte contre l'immigration illégale reste centrée sur le migrant et non sur les caractéristiques des pays d'accueil qui incitent à l'immigration illégale. En effet, le véritable appel d'air pour cette immigration est la tolérance dans les pays occidentaux d'un marché du travail illégal très développé. L'immigrant illégal sait qu'il trouvera à l'arrivée un travail très dur et fortement sous-payé par rapport à la norme du pays d'accueil, mais cependant plus lucratif que dans son pays d'origine. Réduire ce marché en augmentant le nombre d'inspecteurs du travail serait assurément plus efficace que des charters pour dissuader les immigrants illégaux de venir en Europe, mais le travail illégal, et plus généralement l'ensemble des entorses au droit du travail, 
concernent en majorité des nationaux ce qui, vu l'importance des intérêts en jeu, empêche jusqu'à présent de s'attaquer efficacement à cet aspect essentiel du problème.

7 Avec une grande hypocrisie, seule la procédure d'asile est donc décrite comme une fenêtre ouverte permettant d'entrer en Europe, et depuis 20 ans l'arsenal juridique est mobilisé pour la fermer ${ }^{1}$.

\section{Asile et illégalité}

8 L'histoire des charters et l'examen de quelques statistiques de base montrent à quel point accuser la procédure d'asile de développer l'immigration illégale est malhonnête. Il est vrai que plus de $80 \%$ des demandeurs sont déboutés et que ces derniers sont suspectés de rester illégalement sur le territoire. Mais, outre le fait que tous les déboutés ne restent pas, sinon les systèmes dactyloscopiques très coûteux destinés à empêcher les demandes d'asile successives dans plusieurs pays européens n'auraient pas lieu d'être, la demande d'asile ne permet pas l'entrée en France. En effet, les dispositifs mis en place font que l'entrée sur le territoire français au titre de l'asile est très difficile. Cela est même totalement impossible aux frontières terrestres puisque les demandeurs se trouvant déjà sur le territoire d'un autre État européen, et qu'ils ont l'obligation de déposer leur demande dans le premier pays démocratique qu'ils traversent (accords Dublin II entre états membres de l'Union Européenne). Les seules entrées suite à une demande d'asile s'effectuent aux frontières aériennes ou maritimes et en 2005 elles représentent moins de $5 \%$ de l'ensemble des demandes déposées en France. Ainsi environ $95 \%$ de ces demandes sont déposées par des personnes entrées au préalable irrégulièrement. Ce qui signifie que les déboutés appartiennent à à deux cas de figure. Soit il s'agit d'immigrants économiques entrés illégalement dans l'optique de travailler, et qui n'ayant pas réussi à régulariser leur situation par l'asile, restent illégaux. Dans ce cas la procédure d'asile n'a pas généré d'immigrants illégaux, elle les a seulement renvoyés dans leur illégalité originelle. Soit ce sont des personnes entrées illégalement pour fuir des persécutions et restant illégalement une fois déboutées faute d'autres perspectives. Dans ce cas la procédure génère bien des illégaux puisque ces personnes sont venues pour obtenir une protection, mais elle le fait par erreur de jugement ou abandon de sa mission de protection des droits humains.

\section{Faire porter la faute sur les demandeurs}

Dans les pays fiers de leur longue renommée de respect des droits humains, ne pas assurer la protection des demandeurs d'asile contre la persécution est une faute que les gouvernements ne veulent pas assumer. Il importe donc de convaincre l'opinion publique que toute la faute revient aux demandeurs d'asile eux-mêmes. Deux grands types d'arguments sont évoqués. Le premier consiste à affirmer que la plupart des demandes ne sont pas fondées sur des craintes réelles de persécutions. Les premiers détournements importants de la procédure d'asile suite aux charters de Pasqua ont beaucoup aidé à répandre dans les esprits les concepts de "faux réfugiés", de "réfugiés économiques", de demandes "manifestement infondées", ou de "demandes abusives». On ne compte plus les déclarations assimilant tous les demandeurs d'asile à des fraudeurs, même les hauts fonctionnaires en charge du dossier de l'asile n'hésitent pas à forcer le trait. Deux exemples caractéristiques: le 
directeur de l'ofpra en 1998 estime qu'il y a autant de réfugiés parmi les demandeurs d'asile que de pépites d'or dans le flux que charrie la rivière ${ }^{2}$; le président de l'ofpra en 2002 va encore plus loin en accusant les réfugiés reconnus par l'ofpra d'être des fraudeurs «le statut ayant été souvent obtenu à la faveur de déclarations frauduleuses que ne peut prouver l'office $»^{3}$.

10 Aujourd'hui, ces concepts sont si bien ancrés dans les esprits que le directeur actuel de l'Ofpra en arrive à justifier les rejets par leur nombre, «M. Jean-Loup Kuhn-Delforge a confirmé que le taux extrêmement élevé de rejet des demandes d'asile (85\%) attestait l'existence de tentatives de détournement de la procédure $»^{4}$. Bel exemple de logique fallacieuse reposant sur l'argument d'autorité, le directeur de l'ofpra nous dit en substance que ce qui atteste que les demandes sont non-fondées est qu'il les juge non-fondées. L'emploi de ce type de logique pourrait permettre à l'inverse d'affirmer que le taux extrêmement élevé de déboutés non reconduits à la frontière (environ $80 \%$ ) atteste que les rejets sont abusifs!

11 Le deuxième type d'arguments pour faire porter la faute du refus d'asile sur les demandeurs eux-mêmes est plus subtil. À ma connaissance, sa première formalisation date de 1991 et sa logique aboutit aujourd'hui à ce que l'on nomme «l'externalisation de l'asile». Dans un article publié sous le pseudonyme de Jacques François (François, 1991), le directeur de l'Ofpra de l'époque distingue le besoin de protection et le niveau de développement du pays où cette protection est sollicitée. "Un facteur économique puissant, l'écart des niveaux de développement, vient s'ajouter aux raisons qui poussent à l'exil, qui devient non seulement une solution à des conflits, mais aussi un moyen d'accéder à un niveau de développement supérieur." Les exemples sont explicites: "Ainsi l'émigration turque d'origine kurde vers les pays d'Europe occidentale trouve son origine dans la violence exercée, de part et d'autre, dans les provinces à majorité kurde, mais se transforme en émigration économique dés lors que les Kurdes, contraints de se déplacer, préferent, plutôt que de s'installer dans les provinces occidentales de la Turquie, franchir les frontières et se diriger vers l'Allemagne, la Suède ou la France. Il en est de même pour les paysans haïtiens fuyant les abus de pouvoir d'un tonton macoute local et se dirigeant non pas vers Port-au-Prince, mais vers New York, Toronto ou Paris, ou encore les Tamouls de Jaffna préférant la vie en Europe plutôt qu'au Tamil Nadu indien, pourtant plus proche géographiquement et culturellement ».

12 C'est ainsi que tout réfugié fuyant des risques avérés de persécutions se «transforme » en immigré économique dès lors qu'il tente d'obtenir l'asile dans un pays ayant un niveau de développement économique supérieur à celui de son pays d'origine. L'Afrique du Sud de l'apartheid avait inventé le développement séparé, cette conception dirige l'Europe vers l'asile séparé : réfugiés de pays pauvres dans les pays pauvres, réfugiés de pays riches (rares) dans les pays riches.

\section{La suppression de toute possibilité effective d'asile en Europe}

13 Quinze ans après leur publication, ces idées ont fait leur chemin et sont de plus en plus appliquées. La notion de "protection à l'intérieur du pays » figure en bonne place dans la directive européenne sur l'asile de $2004^{5}$ et celle d'asile au plus près des pays d'origine est au centre de la politique actuelle d'endiguement des flux. Après avoir développé les outils juridiques qui ont permis de réduire fortement le nombre de 
demandes d'asile déposées en Europe et de débouter plus de $80 \%$ des demandeurs, la dernière phase de l'endiguement consiste en effet à développer des procédures d'asile formellement respectueuses des droits humains dans les pays limitrophes de l'Union. Suite notamment aux événements de Ceuta et Melilla, l'Europe participe déjà à de tels programmes au Maroc.

Que l'Union apporte une aide financière, matérielle et conceptuelle à la mise en place de procédures d'asile dans des pays où ces procédures sont insuffisamment développées est en soit une très bonne chose. On ne peut que souhaiter que le Maroc, par exemple, se dote de tous les moyens nécessaires pour analyser sereinement les demandes d'asile dans un total respect des conventions internationales et pour protéger efficacement les réfugiés. Penser que ces pays seraient éternellement incapables d'offrir un asile aussi respectueux des droits humains que les pays de l'Union européenne serait faire preuve d'un grand mépris bien proche du racisme colonial.

Dans ce nouveau contexte, la conception classique du lieu d'asile "normal» pose cependant un problème. En effet, selon le HCR «Les gens ont le droit de chercher refuge, mais ils n'ont pas le droit de choisir un lieu d'asile à leur convenance. Ce n'est pas aux requérants de décider comment seront réparties les charges résultant de l'octroi de l'asile. Les réfugiés sont censés demander l'asile dans le premier pays sûr où ils arrivent » (HCR, 1994). La conséquence logique de la mise en place de procédures d'asile respectueuses des droits humains dans tous les pays limitrophes de l'Union est donc la fin de l'asile en Europe puisque toute personne persécutée hors de l'Union trouvera sur sa route ces pays offrant un asile aussi sûr qu'en Europe. La création de ce «cordon sanitaire » supprime de facto la question de l'asile en Europe. Au même titre qu'aucune demande n'est enregistrée aux frontières terrestres françaises puisque nous sommes entourés de pays sûrs, il n'y aura bientôt plus aucune possibilité de déposer une demande aux frontières terrestres extérieures de l'Union.

La question de la demande aux frontières aériennes est déjà quasiment réglée par l'obligation de visa pour les ressortissants des pays d'origine des demandeurs d'asile et l'obligation faite aux compagnies aériennes de vérifier la possession de ces visas avant l'embarquement sous peine d'une amende de 5000 euros par passager démuni des documents de voyage requis. Cette délocalisation des contrôles se perfectionne sans cesse avec l'usage des nouvelles technologies de la communication. À terme, ces dernières devraient permettre un contrôle complet des passagers par les pays de destination avant l'embarquement dans les pays d'origine.

17 Reste la question des arrivées clandestines. L'Union a déjà inventé des outils pour refuser d'examiner les demandes d'asile déposées après une entrée illégale. En vertu du même principe que la demande doit être déposée dans le premier pays sûr traversé, les pays de l'Union ont signé entre eux ${ }^{6}$ puis avec d'autres pays des accords de réadmission des demandeurs d'asile ayant transité sur leur territoire. Ces accords sont cependant d'une application lourde et complexe, il est souvent malaisé d'apporter la preuve qu'un demandeur a transité par un pays voisin. L'Union travaille à améliorer l'efficacité de ces renvois. De nouveaux accords de réadmission, la plupart sans référence au droit d'asile, sont en cours de négociation et concernent de nombreux pays d'émigration (Hongkong, Macao, Albanie, Sri Lanka, Syrie, Maroc, Russie, Chine, Turquie, Ukraine, Moldavie, Roumanie...). On doit également signaler le projet de Tony Blair connu sous le nom d'externalisation de l'asile qui vise à rendre obligatoire le dépôt des demandes à 
partir de camps extérieurs à l'Union et à systématiser le renvoi des demandeurs entrés illégalement.

\section{Sauver l'asile en Europe par la liberté d'immigrer}

18 La suppression de toute possibilité d'asile en Europe est la conséquence logique de la négation de la liberté de choix de son pays d'asile. L'esprit des textes fondateurs de la République : "Le peuple français donne asile aux étrangers bannis de leur patrie pour la cause de la liberté. Il le refuse aux tyrans $\aleph^{7}$ nous conduit pourtant à revendiquer cette liberté pour tous les demandeurs d'asile. Mais la rigueur scientifique nous impose de poser immédiatement la question qui en découle inexorablement: pourquoi un réfugié aurait-il le choix de son pays d'asile alors qu'un immigré économique fuyant la famine ne dispose pas de la liberté d'installation dans le pays de son choix?

Il faut ici dire un mot sur la légitimité de l'accueil des réfugiés en période de fermeture des frontières. Pourquoi une personne craignant avec raison de mourir sous les persécutions est-elle accueillie alors qu'une autre craignant avec raison de mourir de faim ne l'est-elle pas? Y aurait-il une hiérarchie dans la noblesse du danger? Non, même si les combattants de la liberté jouissent d'une aura qui manque aux miséreux, l'explication théorique est ailleurs. Toutes les personnes risquant de mourir doivent être secourues, mais les victimes des famines sont censées pouvoir être secourues sur place, ce qui dispense de devoir les accueillir, alors que les victimes de persécutions ne peuvent l'être sauf à envahir militairement les pays concernés pour y faire régner l'ordre de l'envahisseur. Faute de pouvoir les protéger sur place, les démocraties doivent accepter d'accueillir les victimes de persécutions qui fuient leur pays.

Cependant, un réfugié fuyant des persécutions en Afrique sub-saharienne n'est plus en danger lorsqu'il se trouve au Maroc et peut y obtenir une protection pleine et entière. Dès lors, plus rien ne justifie une différence de traitement avec l'immigré économique et revendiquer le libre choix du pays d'asile doit s'accompagner d'une revendication de la liberté d'installation pour tous.

21 Bien entendu, la liberté totale de l'immigration ne peut être conçue sans développement simultané des droits sociaux dans les pays d'accueil. La liberté d'exploiter des travailleurs libres ramènerait aux sources du capitalisme sauvage du $\mathrm{XIX}^{\text {ème }}$ siècle. La conjoncture politique n'est cependant pas propice à un renforcement du droit du travail. Même si le contrat première embauche a été supprimé de haute lutte, la précarité du contrat nouvelle embauche ou l'abaissement de l'âge à partir duquel les enfants peuvent travailler de nuit ${ }^{8}$ restent d'actualité.

\section{Conclusion}

Les vingt ans écoulés depuis la création de la Revue Européenne des Migrations Internationales ont vu la politique de maitrise de l'immigration supprimer en pratique l'asile en Europe. Face à cet abandon de la défense des droits humains, notamment dans une France qui se glorifie de son passé de patrie des droits de l'homme, la légitimité des contrôles migratoires doit être interrogée. Certes, dès 1789 , la révolution française a établi une distinction entre les droits de l'homme et ceux du citoyen. Les citoyens, collectivement propriétaires du territoire national, jouissent d'un droit de propriété 
déclaré sacré ${ }^{9}$ qui justifie la possibilité d'exclusion des non-citoyens. N'est-il pas temps de réconcilier l'Homme et le citoyen (sans connotation de genre) ? Puisque la citoyenneté européenne en gestation ne fait que reproduire les clivages à une échelle spatiale plus large, est-il préférable d'abandonner la forteresse Europe ou les droits humains?

\section{BIBLIOGRAPHIE}

ANGOUSTURES Aline, LEGOUX Luc, JAGGERS Christophe (1995) Que sont devenus les demandeurs d'asile de 1990 ?, REMI (Revue européenne des migrations internationales), vol. 11, $\mathrm{n}^{\circ} 3$, pp. 105-121.

LEGOUX Luc (1995) La crise de l'asile politique en France, CEPED, 344 p.

LEGOUX Luc (2004) Changements et permanences dans la protection des réfugiés, REMI (Revue européenne des migrations internationales), vol. 20, $\mathrm{n}^{\circ} 2$, pp. 9-22.

TERRAL Jean-François (1998) L'accueil des demandeurs d'asile et des réfugiés, Échanges santésocial, sept 1998, numéro 91 pp. 18-21.

FRANÇOIS Jacques (1991) De l'exil à l'asile en Europe, in Hermant, Daniel, Bigo Didier (dir) Approches Polémologiques, Conflits et violences politiques dans le monde dans les années quatre-vingt-dix, ed. Fondation pour les études de défense nationale/Institut français de polémologie, pp 416-428.

HCR (1994) Les réfugiés dans le monde L'enjeu de la protection, Paris, La découverte, $192 \mathrm{p}$.

\section{NOTES}

1. Voir l'analyse résumée de la logique du système d'asile en Europe de 1945 à nos jours dans Legoux 2004.

2. À propos du métier d'officier de protection de l'ofpra « Métier difficile, qui demande rigueur et motivation, et qui s'apparente à celui d'orpailleur : dans le flux que charrie la rivière, chercher inlassablement les pépites d'or de la convention de Genève, les réfugiés politiques » (Terral, 1998).

3. «Les dérives du droit d'asile en France » Jean-Pierre Lafon, ministre plénipotentiaire, directeur des Français à l'étranger et des étrangers en France, note publiée dans l'Express 10-01-02.

4. Travaux de la commission d'enquête sur l'immigration, audition de M. Jean-Loup KuhnDelforge directeur général de l'Office français de protection des réfugiés et apatrides (OFPRA), http://www.senat.fr/bulletin/20051212/immigration.html)

5. Article 8 de la Directive qualification (droit d'asile) du 29 avril 2004.

6. Accords de Schengen puis convention de Dublin et règlement Dublin II.

7. Article 120 de la constitution de 1793.

8. La loi dite sur l'égalité des chances autorise le travail de nuit des enfants dans le cadre de l'apprentissage à partir de 15 ans au lieu de 16 ans auparavant. 
9. Déclaration des droits de l'homme et du citoyen: Article 2 - Le but de toute association politique est la conservation des droits naturels et imprescriptibles de l'homme. Ces droits sont la liberté, la propriété, la sûreté et la résistance à l'oppression. Article 17 - La propriété étant un droit inviolable et sacré, nul ne peut en être privé, si ce n'est lorsque la nécessité publique, légalement constatée, l'exige évidemment, et sous la condition d'une juste et préalable indemnité.

\section{RÉSUMÉS}

Il y a vingt ans la forte croissance du nombre des demandeurs d'asile transformait la défense des droits humains en problème migratoire. Depuis l'état ne cesse de faire porter la faute du refus de protection sur les demandeurs eux-mêmes qui se transformeraient en immigrés économiques illégaux dés lors qu'ils cherchent asile dans un pays économiquement plus développé que leur pays d'origine. La mise en place d'un « cordon sanitaire » de pays sûrs autour de l'Union où les réfugiés sont censés devoir trouver une protection pose la question de l'alternative entre la fin de l'asile en Europe et la liberté de choix de son pays d'asile. Par extension cette question pose celle de la liberté totale d'immigration et du développement simultané des droits sociaux dans les pays d'accueil.

Asylum and immigration : reconciling human and citizens rights. Twenty years ago the sharp increase in the number of asylum seekers transformed the defence of human rights into a problem linked to migration flows. Since then, governments of receiving countries constantly refuse to grant asylum arguing that asylum seekers are in fact undocumented economic migrants looking for better economic opportunities. The setting up of a "sanitary belt" in reliable countries surrounding the UE, where individuals are supposed to be politically safe shows that we are faced with a dilemna: how to reconcile the fact that we are witnessing the end of a period when Europe was a region offering political asylum and the individual's freedom of choice of the country of asylum. This issue raises another one, that of the total freedom of immigration and of the recognition of social rights in receiving countries.

Asilo, inmigración: reconciliar los derechos del hombre y los del ciudadano. Hace veinte años el fuerte crecimiento del número de demandantes de asilo transformaba la defensa de los derechos humanos en un problema migratorio. Desde entonces, el Estado deniega la protección a los demandantes acusándoles de ser inmigrantes económicos por buscar asilo en países económicamente más desarrollados que sus países de origen. La creación de un "cordón sanitario» de países seguros alrededor de la Unión, donde los refugiados deberían encontrar protección, pone sobre la mesa la cuestión de la alternativa entre el fin del asilo en Europa y la libertad de elección del país de asilo. Por extensión, esta cuestión conduce a otra, la de la libertad total de inmigración y del desarrollo simultaneo de los derechos sociales en los países de acogida.

\section{INDEX}

Mots-clés : asile politique, demandeurs d'asile, droit d'asile 
AUTEUR

LUC LEGOUX

Maître de conférences, Institut de démographie de l'Université Paris I, 90 rue de Tolbiac, 75013

Paris, courriel legoux@univ-Paris1.fr 